\title{
Coulomb's law corrections from a gauge-kinetic mixing
}

\author{
Patricio Gaete* and Iván Schmidt \\ Departmento de Física and Centro Científico-Tecnológico de Valparaíso, \\ Universidad Técnica Federico Santa María, Valparaíso, Chile \\ November 11, 2018
}

\begin{abstract}
We study the static quantum potential for a gauge theory which includes the mixing between the familiar photon $U(1)_{Q E D}$ and a second massive gauge field living in the so-called hidden-sector $U(1)_{h}$. Our discussion is carried out using the gauge-invariant but path-dependent variables formalism, which is alternative to the Wilson loop approach. Our results show that the static potential is a Yukawa correction to the usual static Coulomb potential. Interestingly, when this calculation is done inside a superconducting box, the Coulombic piece disappears leading to a screening phase.
\end{abstract}

PACS numbers: 11.15.-q; 11.10.Ef; 11.30.Cp

Keywords: gauge invariance, static potential, gauge-kinetic mixing

The formulation and experimental consequences of extensions of the Standard Model (SM), such as axion-like particles and light extra hidden $U(1)$ gauge bosons have been extensively discussed in the last few years, in order to explain cosmological and astrophysical results [1]. In this case, hidden sector refers to a set of so far unobserved degrees of freedom very weakly coupled to the SM. We further note that the existence of weakly interacting particles, which couple to the electromagnetic sector, also are of interest due to their appearance in string theories physics. Nevertheless, although none of these searches ultimately has yielded a positive signal, the arguments in favor of the existence of axion-like particles or light extra hidden $U(1)$ gauge bosons, remain a relevant and challenging issue.

It is worthy recalling at this stage that the axion-like scenario can be qualitatively understood by the existence of light pseudo-scalar bosons $\phi$ ("axions"), with a coupling to two photons. As a consequence, the corresponding term in the effective Lagrangian has the form $\mathcal{L}_{I}=-1 / 4 M F_{\mu \nu} \widetilde{F}^{\mu \nu} \phi$, where $\widetilde{F}^{\mu \nu}=1 / 2^{\varepsilon_{\mu \nu \lambda \rho}} F^{\lambda \rho}$. We also recall that axionic electrodynamics experiences mass generation due to the breaking of rotational invariance induced by a classical background configuration of the gauge field strength [2], and leads to confining potentials in the presence of nontrivial constant expectation values for the gauge field strength $F_{\mu \nu}$ [3]. In fact, in the case of a constant electric field strength expectation value, the static potential remains Coulombic, while in the case of a constant magnetic field strength expectation value the potential energy is the sum of a Yukawa and a linear potential, leading to the confinement of static charges. Another interesting observation is that the magnetic character of the field strength expectation value needed to obtain confinement is in agreement with the current chromo-magnetic picture of the $Q C D$ vacuum

\footnotetext{
* patricio.gaete@usm.cl

$\dagger$ ivan.schmidt@usm.cl
}

[4 6]. In addition, similar results have been obtained in the context of the dual Ginzburg-Landau theory [7], as well as for a theory of antisymmetric tensor fields that results from the condensation of topological defects as a consequence of the Julia-Toulouse mechanism [8].

As already expressed, the extra hidden $U(1)$ gauge bosons scenario, which is described by the mixing between the familiar photon $U(1)_{Q E D}$ and a second gauge field (paraphoton) living in the hidden-sector $U(1)_{h}$, has been inspired by studies coming from the realms of string theory [9, 10], as well as from quantum field theory [1118]. Actually, as mentioned in [19], the introduction of a second gauge field in addition to the usual photon was pioneered in Ref. [20], in the context of electrodynamics in the presence of magnetic monopoles [21]. The quantization for a system with two photons was later carried out in [22]. Let us also mention here that the possible existence of massive vector fields was also proposed in [23]. Most recently, a kinetic term between the familiar photon $U(1)_{Q E D}$ and a second gauge field has been considered in order to explain recent unexpected observations in high energy astrophysics [24].

Given the ongoing experiments related to this type of physics [25 28], it should be interesting to acquire a better understanding what might be the observational signatures presented by the hidden-sector $U(1)_{h}$. Hence, our purpose here is to investigate the impact of paraphotons on physical observables, in particular the static potential between two charges, using the gauge-invariant but path-dependent variables formalism, which is alternative to the Wilson loop approach. Of special interest will be to explore the existence of duality (equivalence) between axion-like particles and light extra hidden $U(1)$ gauge bosons models, by comparing physical quantities in both theories.

We now proceed to analyze the interaction energy between static point-like sources for the model under consideration. The main tool in our analysis will be to compute the expectation value of the energy operator $H$ in the physical state $|\Phi\rangle$ describing the sources, which we will denote by $\langle H\rangle_{\Phi}$. 
As we have already pointed out, the gauge theory we are considering describes the coupling between the familiar massless electromagnetism $U(1)_{Q E D}$ and a hiddensector $U(1)_{h}$. In this case the corresponding theory is governed by the Lagrangian density [29]:

$$
\mathcal{L}=-\frac{1}{4} F_{\mu \nu} F^{\mu \nu}-\frac{1}{4} B_{\mu \nu} B^{\mu \nu}-\frac{1}{2} \chi F^{\mu \nu} B_{\mu \nu}+\frac{1}{2} m_{\gamma^{\prime}}^{2} B_{\mu} B^{\mu},
$$

where $\chi$ is a dimensionless parameter. Recalling again that the theory we are considering emerges naturally in extensions of the Standard Model in the context of string theories with nonvanishing values for $\chi$ [9, 30]. We also note here that in expression (1) the first two terms are the standard kinetic terms for the photon and hidden-sector photon fields, respectively. The third term is the so called gauge-kinetic mixing, while the last term accounts for a possible mass of the paraphoton, which may arise via a Higgs or Stüeckelberg mechanism. After shifting the $B_{\mu^{-}}$ field as $B_{\mu} \longrightarrow \tilde{B}_{\mu}-\chi A_{\mu}$ in (1), we obtain an equivalent Lagrangian density:

$$
\begin{aligned}
\mathcal{L} & =-\frac{1}{4}\left(1-\chi^{2}\right) F_{\mu \nu} F^{\mu \nu}+\frac{1}{2} \chi^{2} m_{\gamma^{\prime}}^{2} A_{\mu} A^{\mu} \\
& +\frac{1}{2} \tilde{B}^{\mu}\left[\eta_{\mu \nu}\left(\Delta+m_{\gamma^{\prime}}^{2}\right)-\partial_{\mu} \partial_{\nu}\right] \tilde{B}^{\nu} \\
& -\chi m_{\gamma^{\prime}}^{2} A_{\mu} \tilde{B}^{\mu} .
\end{aligned}
$$

Next, by integrating out the $\tilde{B}_{\mu}$-field in the above expression, one gets an effective action for the $A_{\mu}$-field that includes the effects of the hidden-sector photon field:

$$
\mathcal{L}_{e f f}=-\frac{1}{4} F_{\mu \nu}\left(1-\chi^{2} \frac{\Delta}{\left(\Delta+m_{\gamma^{\prime}}^{2}\right)}\right) F^{\mu \nu} .
$$

Let us also mention here that in addition to the above transformation to diagonalize the kinetic mixing term, we can also choose the transformation $A_{\mu} \longrightarrow \tilde{A}_{\mu}-\chi B_{\mu}$, $\tilde{B}_{\mu}=B_{\mu}$. As a consequence, there is no kinetic mixing between $\tilde{A}_{\mu}$ and $\tilde{B}_{\mu}$, and one obtains two noninteracting gauge theories with a trivial vacuum. In this perspective, the transformation leading to (3) induces a richer dynamics that incorporate the effects of the hidden-sector photon field. In other words, this analysis renders manifest the signatures of the hidden-sector $U(1)_{h}$ on the ordinary photon sector.

Having characterized the theory under study, we can now compute the interaction energy. To this end, we shall first examine the Hamiltonian framework for this theory. The canonical momenta $\Pi^{\mu}$, conjugate to $A^{\mu}$, are found to be $\Pi^{0}=0$, which is the primary constraint, while the other momenta components are $\Pi^{i}=-\frac{\left(1-\chi^{2}\right) \Delta+m_{\gamma^{\prime}}^{2}}{\left(\Delta+m_{\gamma^{\prime}}^{2}\right)} F^{0 i}$. The canonical Hamiltonian is now obtained in the usual way

$H_{C}=\int d^{3} x\left\{-A^{0} \partial_{i} \Pi^{i}+\frac{1}{2\left(1-\chi^{2}\right)} \Pi^{i} \frac{\left(\Delta+m_{\gamma^{\prime}}^{2}\right)}{\left(\Delta+M_{\gamma^{\prime}}^{2}\right)} \Pi^{i}\right\}$

$$
+\int d^{3} x\left\{\frac{\left(1-\chi^{2}\right)}{4} B^{i} \frac{\left(\Delta+M_{\gamma^{\prime}}^{2}\right)}{\left(\Delta+m_{\gamma^{\prime}}^{2}\right)} B^{i}\right\}
$$

where $M_{\gamma^{\prime}}^{2} \equiv m_{\gamma^{\prime}}^{2} /\left(1-\chi^{2}\right)$, and $B^{i}$ is the magnetic field. Persistency of the primary constraint in time yields a secondary constraint, which is the usual Gauss constraint $\Gamma_{1}(x) \equiv \partial_{i} \Pi^{i}=0$, and time stability of this secondary constraint does not induce further constraints. Therefore, the extended Hamiltonian that generates translations in time reads $H=$ $H_{C}+\int d^{3} x\left(c_{0}(x) \Pi_{0}(x)+c_{1}(x) \Gamma_{1}(x)\right)$. Here $c_{0}(x)$ and $c_{1}(x)$ are arbitrary Lagrange multipliers. Moreover, it is straightforward to see that $\dot{A}_{0}(x)=\left[A_{0}(x), H\right]=c_{0}(x)$, which is an arbitrary function. Since $\Pi^{0}=0$ always, neither $A^{0}$ nor $\Pi^{0}$ are of interest in describing the system and may be discarded from the theory. If a new arbitrary coefficient $c(x)=c_{1}(x)-A_{0}(x)$ is introduced the Hamiltonian may be rewritten in the form

$$
\begin{aligned}
H & =\int d^{3} x\left\{c(x) \partial_{i} \Pi^{i}+\frac{1}{2\left(1-\chi^{2}\right)} \Pi^{i} \frac{\left(\Delta+m_{\gamma^{\prime}}^{2}\right)}{\left(\Delta+M_{\gamma^{\prime}}^{2}\right)} \Pi^{i}\right\} \\
& +\int d^{3} x\left\{\frac{\left(1-\chi^{2}\right)}{4} B^{i} \frac{\left(\Delta+M_{\gamma^{\prime}}^{2}\right)}{\left(\Delta+m_{\gamma^{\prime}}^{2}\right)} B^{i}\right\} .
\end{aligned}
$$

In accordance with the Dirac method, we must fix the gauge. A particularly convenient gauge fixing condition is

$$
\Gamma_{2}(x) \equiv \int_{C_{\xi x}} d z^{\nu} A_{\nu}(z) \equiv \int_{0}^{1} d \lambda x^{i} A_{i}(\lambda x)=0,
$$

where $\lambda(0 \leq \lambda \leq 1)$ is the parameter describing the spacelike straight path $x^{i}=\xi^{i}+\lambda(x-\xi)^{i}$, and $\xi$ is a fixed point (reference point). There is no essential loss of generality if we restrict our considerations to $\xi^{i}=0$. The choice (6) leads to the Poincaré gauge [31, 32]. With this, we arrive at the only nonvanishing equal-time Dirac bracket for the canonical variables

$$
\begin{aligned}
\left\{A_{i}(x), \Pi^{j}(y)\right\}^{*} & =\delta_{i}^{j} \delta^{(3)}(x-y) \\
& -\partial_{i}^{x} \int_{0}^{1} d \lambda x^{j} \delta^{(3)}(\lambda x-y) .
\end{aligned}
$$

Having thus obtained the quantization of the model under consideration, we are now in a position to compute the interaction energy. As already expressed, to this end we will work out the expectation value of the energy operator $H$ in the physical state $|\Phi\rangle$, which has the important property of being gauge-invariant [33, 34], and has the stringy form

$$
\begin{aligned}
|\Phi\rangle & \equiv\left|\bar{\Psi}(\mathbf{y}) \Psi\left(\mathbf{y}^{\prime}\right)\right\rangle \\
& =\bar{\psi}(\mathbf{y}) \exp \left(i q \int_{\mathbf{y}^{\prime}}^{\mathbf{y}} d z^{i} A_{i}(z)\right) \psi\left(\mathbf{y}^{\prime}\right)|0\rangle,
\end{aligned}
$$


where $|0\rangle$ is the physical vacuum state and the line integral appearing in the above expression is along a spacelike path starting at $\mathbf{y}$ ' and ending at $\mathbf{y}$, on a fixed time slice.

Taking into account the above Hamiltonian structure, we observe that

$$
\begin{aligned}
\Pi_{i}(x)\left|\bar{\Psi}(\mathbf{y}) \Psi\left(\mathbf{y}^{\prime}\right)\right\rangle & =\bar{\Psi}(\mathbf{y}) \Psi\left(\mathbf{y}^{\prime}\right) \Pi_{i}(x)|0\rangle \\
& +q \int_{\mathbf{y}}^{\mathbf{y}^{\prime}} d z_{i} \delta^{(3)}(\mathbf{z}-\mathbf{x})|\Phi\rangle .
\end{aligned}
$$

Having made this observation and since the fermions are taken to be infinitely massive (static) we can substitute $\Delta$ by $-\nabla^{2}$ in Eq. (5). Therefore, the expectation value $\langle H\rangle_{\Phi}$ is expressed as

$$
\langle H\rangle_{\Phi}=\langle H\rangle_{0}+\langle H\rangle_{\Phi}^{(1)}+\langle H\rangle_{\Phi}^{(2)},
$$

where $\langle H\rangle_{0}=\langle 0|H| 0\rangle$. The $\langle H\rangle_{\Phi}^{(1)}$ and $\langle H\rangle_{\Phi}^{(2)}$ terms are given by

$$
\langle H\rangle_{\Phi}^{(1)}=-\frac{1}{2}\left\langle\Phi\left|\int d^{3} x \Pi_{i} \Pi^{i}\right| \Phi\right\rangle,
$$

and

$$
\langle H\rangle_{\Phi}^{(2)}=-\frac{1}{2} \frac{\chi^{2}}{\left(1-\chi^{2}\right)}\left\langle\Phi\left|\int d^{3} x \Pi_{i} \frac{\nabla^{2}}{\left(\nabla^{2}-M_{\gamma^{\prime}}^{2}\right)} \Pi^{i}\right| \Phi\right\rangle .
$$

Following our earlier procedure [35, 36], we see that the potential for two opposite charges, located at $\mathbf{y}$ and $\mathbf{y}^{\prime}$, takes the form

$$
V=-\frac{q^{2}}{4 \pi}\left(\frac{1}{L}+\frac{\chi^{2}}{\left(1-\chi^{2}\right)} \frac{e^{-M_{\gamma^{\prime}} L}}{L}\right),
$$

where $\left|\mathbf{y}-\mathbf{y}^{\prime}\right| \equiv L$. We stress that the choice of the gauge is in this approach really arbitrary. Being the formalism completely gauge invariant, we would obtain exactly the same result in any gauge.

It is worth noting here that there is an alternative but equivalent way of obtaining the result (13), which highlights certain distinctive features of our methodology. We start by considering [35]:

$$
V \equiv q\left(\mathcal{A}_{0}(\mathbf{0})-\mathcal{A}_{0}(\mathbf{y})\right),
$$

where the physical scalar potential is given by

$$
\mathcal{A}_{0}\left(x^{0}, \mathbf{x}\right)=\int_{0}^{1} d \lambda x^{i} E_{i}(\lambda \mathbf{x}),
$$

with $i=1,2,3$. This follows from the vector gaugeinvariant field expression 31]

$$
\mathcal{A}_{\mu}(x) \equiv A_{\mu}(x)+\partial_{\mu}\left(-\int_{\xi}^{x} d z^{\mu} A_{\mu}(z)\right),
$$

where, as in Eq.(6), the line integral is along a spacelike path from the point $\xi$ to $x$, on a fixed slice time.
The gauge-invariant variables (16) commute with the sole first constraint (Gauss' law), confirming that these fields are physical variables 21]. Note that Gauss' law for the present theory reads $\partial_{i} \Pi^{i}=J^{0}$, where we have included the external current $J^{0}$ to represent the presence of two opposite charges. For $J^{0}(t, \mathbf{x})=q \delta^{(3)}(\mathbf{x})$ the electric field is given by

$$
E^{i}=q \partial^{i}\left(G(\mathbf{x})+\frac{\chi^{2}}{\left(1-\chi^{2}\right)} \tilde{G}(\mathbf{x})\right),
$$

where $G(\mathbf{x})=\frac{1}{4 \pi} \frac{1}{|\mathbf{x}|}$ and $\tilde{G}(\mathbf{x})=\frac{e^{-M_{\gamma^{\prime}}|\mathbf{x}|}}{4 \pi|\mathbf{x}|}$ are the Green functions in three space dimensions. Finally, replacing this result in (15) and using (14), the potential for a pair of point-like opposite charges q located at $\mathbf{0}$ and $\mathbf{L}$ becomes

$$
V=-\frac{q^{2}}{4 \pi}\left(\frac{1}{L}+\frac{\chi^{2}}{\left(1-\chi^{2}\right)} \frac{e^{-M_{\gamma^{\prime} L}}}{L}\right),
$$

where $|\mathbf{L}| \equiv L$.

Mention should be made, at this point, about a new and simple experiment for searching for hidden-sector $U(1)$ gauge bosons with gauge kinetic mixing with the ordinary photon [37]. The crucial idea underlying this suggestion consists in putting a sensitive magnetometer inside a superconducting shielding, which in turn is placed inside a strong magnetic field. As a result it was argued that photon - hidden photon oscillations would allow to penetrate the superconductor and a magnetic field would register on the magnetometer, in contrast with the usual electrodynamics where the magnetic field cannot penetrate the superconductor. For this purpose the authors of Ref. 37] consider the Lagrangian density:

$$
\begin{aligned}
\mathcal{L} & =-\frac{1}{4} F_{\mu \nu} F^{\mu \nu}-\frac{1}{4} B_{\mu \nu} B^{\mu \nu}-\frac{1}{2} \chi F^{\mu \nu} B_{\mu \nu}+\frac{1}{2} m_{\gamma^{\prime}}^{2} B_{\mu} B^{\mu} \\
& +\frac{1}{2} M_{L o n}^{2} A_{\mu} A^{\mu},
\end{aligned}
$$

where the last term corresponds to the London mass $\left(M_{L o n}\right)$ that the photon acquires inside the superconductor. Notice that in vacuum $M_{\text {Lon }}=0$, and expression (19) reduces to expression (11). Given its relevance, it is of interest to study the stability of the above scenario (19) for the $M_{\text {Lon }} \neq 0$ case.

Following the same steps that lead to (10) we obtain

$$
\begin{aligned}
\langle H\rangle_{\Phi}^{(1)} & =\frac{1}{2} \frac{1}{\left(1-\chi^{2}\right)} \frac{\left(M_{1}^{2}-m_{\gamma^{\prime}}^{2}\right)}{\left(M_{2}^{2}-M_{1}^{2}\right)} \\
& \times\left\langle\Phi\left|\int d^{3} x \Pi_{i} \frac{\nabla^{2}}{\left(\nabla^{2}-M_{1}^{2}\right)} \Pi^{i}\right| \Phi\right\rangle,
\end{aligned}
$$

and

$$
\begin{aligned}
\langle H\rangle_{\Phi}^{(2)} & =-\frac{1}{2} \frac{1}{\left(1-\chi^{2}\right)} \frac{\left(M_{2}^{2}-m_{\gamma^{\prime}}^{2}\right)}{\left(M_{2}^{2}-M_{1}^{2}\right)} \\
& \times\left\langle\Phi\left|\int d^{3} x \Pi_{i} \frac{\nabla^{2}}{\left(\nabla^{2}-M_{2}^{2}\right)} \Pi^{i}\right| \Phi\right\rangle
\end{aligned}
$$


where

$$
M_{1}^{2}=\frac{\left(m_{\gamma^{\prime}}^{2}+M_{L o n}^{2}\right)^{2}}{2\left(1-\chi^{2}\right)^{2}}\left[1+\sqrt{1-4\left(1-\chi^{2}\right)^{3} \frac{m_{\gamma^{\prime}}^{2} M_{L o n}^{2}}{\left(m_{\gamma^{\prime}}^{2}+M_{L o n}^{2}\right)^{4}}}\right]
$$

and

$$
M_{2}^{2}=\frac{\left(m_{\gamma^{\prime}}^{2}+M_{L o n}^{2}\right)^{2}}{2\left(1-\chi^{2}\right)^{2}}\left[1-\sqrt{1-4\left(1-\chi^{2}\right)^{3} \frac{m_{\gamma^{\prime}}^{2} M_{L o n}^{2}}{\left(m_{\gamma^{\prime}}^{2}+M_{L o n}^{2}\right)^{4}}}\right] .
$$

Again, following our earlier procedure [35, 36], we see that the potential for two opposite charges, located at $\mathbf{y}$ and $\mathbf{y}^{\prime}$, takes the form

$$
\begin{aligned}
V & =-\frac{q^{2}}{4 \pi} \frac{1}{\left(1-\chi^{2}\right)\left(M_{2}^{2}-M_{1}^{2}\right)} \\
& \times\left[\left(M_{2}^{2}-m_{\gamma^{\prime}}^{2}\right) \frac{e^{-M_{2} L}}{L}-\left(M_{1}^{2}-m_{\gamma^{\prime}}^{2}\right) \frac{e^{-M_{1} L}}{L}\right] .
\end{aligned}
$$

Hence we see that for $M_{\text {Lon }}=0$, expression (22) reduces to expression (13). Therefore, when the analysis is done inside a superconducting box, the surprising result is that the theory describes an exactly screening phase.

To conclude, once again we have exploited a crucial point for understanding the physical content of gauge theories, that is, the identification of field degrees of freedom with observable quantities. Our analysis reveals that the static potential profile obtained from both the coupling between the familiar massless electromagnetism $U(1)_{Q E D}$ and a hidden-sector $U(1)_{h}$ and axionic electrodynamics models are quite different. This means that the two theories are not equivalent. As it was shown in [3], axionic electrodynamics has a different structure which is reflected in a confining piece, which is not present in the coupling between the familiar massless electromagnetism $U(1)_{Q E D}$ and a hidden-sector $U(1)_{h}$ scenario.

This work was partially supported by Fondecyt (Chile), projects 1080260 and 1100287.
[1] E. Zavattini et al. [PVLAS collaboration], Phys. Rev. Lett. 96, 110406 (2006); R. Cameron et al. [BFRT collaboration], Phys. Rev. D47, 3707 (1993); S. J. Chen et al. [Q and A collaboration], Mod. Phys. Lett. A22, 2815 (2007);Zavattini2 E. Zavattini et al. [PVLAS collaboration], Phys. Rev. D77, 032006 (2008); C. Robilliard et al. [BMV collaboration], Phys. Rev. Lett. 99, 190403 (2007); A. S. Chou et al. [Gamme V (T-969) collaboration], Phys. Rev. Lett. 100, 080402 (2008); M. Ahlers, H. Gies, J. Jaeckel, J. Redondo and R. Ringwald, Phys. Rev. D77, 095001 (2008); M. Ahlers, H. Gies, J. Jaeckel, J. Redondo and R. Ringwald, Phys. Rev. D76, 115005 (2007); V. Popov, Tr. J. of Physics, 23, 943 (1999); V. Popov and O. Vasil'ev, Europhys. Lett. 15, 7 (1991); D. Chelouche et al., Astrophys. J. Suppl. 1801 (2009); S. N. Gninenko, arXiv: 0802.1315 [hep-ph].

[2] S. Ansoldi, E. I. Guendelman and E. Spallucci, JHEP 09, 044 (2003).

[3] P. Gaete and E. I. Guendelman, Mod. Phys. Lett. A20, 319 (2005).

[4] G. K. Savvidy, Phys. Lett. B71, 133 (1977).

[5] H.B. Nielsen and M. Ninomiya, Nucl. Phys. B156, 1 (1979).

[6] H. B. Nielsen and P. Olesen, Nucl. Phys. B160, 380 (1979).

[7] H. Suganuma, S. Sasaki and H. Toki, Nucl. Phys. B435, 207 (1995).

[8] P. Gaete and C. Wotzasek, Phys. Lett. B601, 108 (2004).

[9] S. A. Abel, M. D. Goodsell, J. Jaeckel, V. V. Khoze and R. Ringwald, JHEP 0807, 124 (2008).

[10] B. Batell and T. Gherghetta, Phys. Rev. D73, 045016 (2006).

[11] B. Holdom, Phys. Lett. B166, 196 (1986).

[12] D. Feldman, Z. Liu and P. Nath, Phys. Rev. D75, 115001 (2007).

[13] B. Körs and P. Nath, Phys. Lett. B586, 366 (2004).

[14] P. Castelo Ferreira, Phys. Lett. B651, 74 (2007).
[15] E. Massó and J. Redondo, JCAP 0509, 015 (2005).

[16] R. Foot, A. Yu. Ignatiev and R. R. Volkas, Phys. Lett. B503, 355 (2001).

[17] D. Singleton, Int. J. Theor. Phys. 34, 37 (1995).

[18] P. Langacker, arXiv: 0801.1345 [hep-ph].

[19] D. Singleton, Am. J. Phys. 64, 452 (1996).

[20] N. Cabibbo and E. Ferrari, Nuovo Cimento 23, 1147 (1962).

[21] P. A. M. Dirac, Proc. R. Soc. A133, 60 (1931); Phys. Rev. 74, 817 (1948).

[22] C. R. Hagen, Phys. Rev. 140, B804 (1965).

[23] L. B. Okun, Sov. Phys. JETP 56, 502 (1982).

[24] N. Arkani-Hamed, D. P. Finkbeiner, T. R. Slatyer and N. Weiner, Phys. Rev. D79 015014 (2009).

[25] K. Ehret et al. [ALPS collaboration], arXiv: hep-ex/0702023

[26] A. V. Afanasev et al., arXiv:hep-ph/0605250

[27] P. Pugnat et al. [OSQAR collaboration], Report N. CERN-SPSC-2006-035.

[28] G. Cantatore [PLVAS collaboration], 3rd Joint ILIASCERN-DESY Axion-WIMPs Training Workshop, 2007, University of Patras, Greece; http://axion-wimp.desy.de

[29] J. Jaeckel and A. Ringwald, arXiv:1002.0329 [hep-ph].

[30] K. Dienes, C. Kolda and J. March-Russell, Nucl. Phys. B492 104 (1997).

[31] P. Gaete, Z. Phys. C76, 355 (1997).

[32] P. Gaete and I. Schmidt, Phys. Rev. D61, 125002 (2000); Phys. Rev. D64, 027702 (2001).

[33] P. A. M. Dirac, The Principles of Quantum Mechanics (Oxford University Press, Oxford, 1958).

[34] P. A. M. Dirac, Can. J. Phys. 33, 650 (1955).

[35] P. Gaete, Phys.Rev. D59, 127702, (1999).

[36] P. Gaete and I. Schmidt, Phys. Rev. D76, 027702 (2007).

[37] J. Jaeckel and J. Redondo, Europhys. Lett. 831002 (2008). 